\title{
Correction to: Multifunctional decoration of alpha-tocopheryl succinate-based NP for cancer treatment: effect of TPP and LTVSPWY peptide
}

\author{
Raquel Palao-Suay ${ }^{1,2}$ - María Rosa Aguilar $\mathbb{D}^{1,2} \cdot$ Francisco J. Parra-Ruiz ${ }^{1,2}$ •

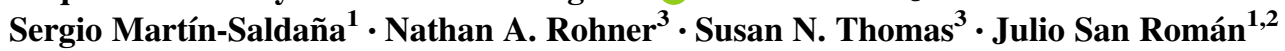

Published online: 12 October 2017

(C) Springer Science+Business Media, LLC 2017

\section{Correction to: J Mater Sci: Mater Med (2017) DOI: 10.1007/s10856-017-5963-y}

The original version of this article unfortunately contained mistakes. The "Reflections on Career Goals" and the group photo were missing in the original version of this article. They are given below. The original article was corrected.

\section{Dr. Maria Rosa Aguilar-Reflections on career goals}

Every goal I have achieved has been possible in the frame of a large family with complementary skills both personally and professionally. Most of my career has been carried out in the Biomaterials Group of the Institute of Polymer Science and Technology (CSIC) and CIBER-BBN in Spain. I have the honor to work with Prof. Julio San Román from whom I learnt about Polymeric Biomaterials and the generation of scientific knowledge for the development of technologies and products of interest to the Biomedical field.

I worked in USA (with Prof. Allan Hoffman), UK (with Prof. Lucy Di Silvio in London and Prof. Andrew Lloyd,

The original article can be found online at https://doi.org/10.1007/ s10856-017-5963-y.

María Rosa Aguilar

mraguilar@ictp.csic.es

1 Group of Biomaterials, Department of Polymeric Nanomaterials and Biomaterials, Institute of Polymer Science and Technology, ICTP-CSIC, Juan de la Cierva, 3, 28006 Madrid, Spain

2 Networking Biomedical Research Centre in Bioengineering, Biomaterials and Nanomedicine, CIBER-BBN, Madrid, Spain

3 George W. Woodruff School of Mechanical Engineering and Parker H. Petit Institute of Bioengineering and Bioscience, Georgia Institute of Technology, 315 Ferst Dr NW, Atlanta 30332 GA, USA
Prof. Matteo Santin, and Prof. Sergey Mikhalovsky in Brighton) and France (with Prof. Françoise Ehrburger-Dolle and Dr. Isabelle Morfin). I would like to thank all these investigators and their teams their shared knowledge and opportunities in the past and present. We also collaborate with multiple institutions and researchers of completely different disciplines that enrich and complement our daily investigation, as the multicentric research shown in this article.

The present work shows our effort to improve cancer treatments and reduce its side effects through the use of multitargeting nanoparticles. This is one of a series of articles based on amphiphilic copolymers bearing $\alpha$-tocopheryl succinate as bioactive molecule. The already described good results using this particular technology have generated commercial interest in a pharmaceutical company. A clinical trial to test the effect of similar nanoparticles to avoid cisplatininduced ototoxicity will start in the following months.

I would like to strengthen our public-private collaboration to facilitate the transfer of our future know-how in shorter periods of time, working hand-by-hand with specialized companies or maybe creating our own spin-off.

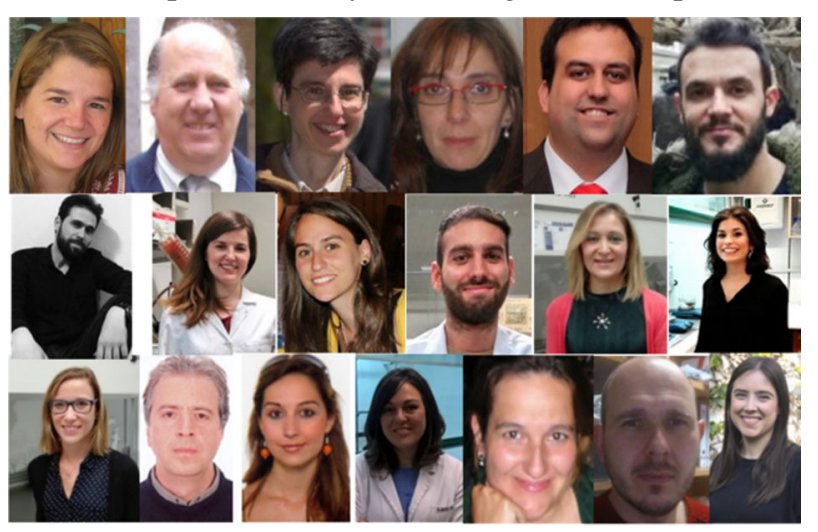

